\title{
Photobiomodulation for the palliation of oral mucositis in cancer patients: the future is here
}

\author{
Yehuda Zadik*,1 \\ ${ }^{1}$ Department of Oral Medicine, Oral \& Maxillofacial Center, Medical Corps, Israel Defense Forces; \& Oral Medicine Clinic for \\ Hematologic \& Oncologic Patients, Department of Oral Medicine, Sedation \& Maxillofacial Imaging, Hebrew University-Hadassah \\ School of Dental Medicine, Jerusalem 9112102, Israel \\ *Author for correspondence: Tel.: +972 52 2385675; Fax: +972 2 6411114; Yehuda.Zadik@mail.huji.ac.il
}

First draft submitted: 1 August 2019; Accepted for publication: 5 September 2019; Published online: 25 October 2019 Keywords: cancer $\bullet$ guidelines $\bullet$ laser $\bullet$ low level laser therapy $\bullet$ oral medicine $\bullet$ oral mucositis $\bullet$ oral oncology $\bullet$
pain $\bullet$ photobiomodulation

For thousands of years, mankind has known about the healing properties of sunlight. However, it was not until the 1960s-70s, where wound healing was shown to be stimulated by low-dose laser treatment, that the term 'photobiostimulation' was coined by Endre Mester. Recently, an increase in our understanding of light therapy has shown that it not only stimulates, but can modify deleterious processes. As a result, photobiomodulation has become acceptable as the collective term for all nonionizing light therapies; including lasers, light emitting diodes and broadband visible light (only low, nonthermal doses in the visible and near-infrared spectrum). These light sources activate endogenous chromophores, eliciting photo-physical and -chemical reactions with favorable clinical therapeutic outcomes such as: promotion of wound healing, tissue regeneration and immune responses; and reduction of negative tissue processes such as inflammation, pain and aberrant immune responses [1]. As such, photobiomodulation has been used for the prevention and treatment of various complications of oncologic treatments, including oral mucositis, radiation dermatitis, dysphagia, hyposalivation and xerostomia (dry mouth), dysgeusia, trismus, osteonecrosis of the jaw (due to radiation therapy or antiresorptive medications), head and neck lymphedema and voice/speech alterations (due to local inflammation) [2].

\section{Oral mucositis}

Oral mucositis causes tremendous suffering in patients treated with radiation to the head and neck, or with certain chemotherapeutics, especially before hematopoietic stem cell transplantation. Oral mucositis manifests as extensive painful ulcers in the oral mucosae. Severe pain from oral mucositis may limit the patients to a liquid-only diet and when oral alimentation is impossible, feeding via percutaneous endoscopic gastrostomy is needed (World Health Organization's grades 3 and 4, respectively) [3,4]. Because of the severe pain and the restricted alimentation, oral mucositis is a major dose-limiting adverse effect (i.e., requiring a dose reduction or premature cessation of the oncologic therapy), prohibiting the patient from receiving optimal anticancer therapy, with obvious influence on the prognosis of the patient. Many interventions have been studied for the prevention and treatment of oral mucositis [5]. The Mucositis Study Group of the Multinational Association of Supportive Care in Cancer (MASCC) and the International Society of Oral Oncology (ISOO) publish and update comprehensive evidence-based clinical practice guidelines for mucositis; these guidelines include a section on photobiomodulation [6,7].

\section{Guidelines}

Recently, a new set of MASCC/ISOO guidelines was published [8]. After a meticulous systematic review of the peer-reviewed literature, and establishment of the Level of evidence for each intervention, the panel recommend intra-oral photobiomodulation therapy, using low-level laser therapy, for the prevention of oral mucositis in specific patient groups. Namely, adult patients receiving hematopoietic stem cell transplantation conditioned with highdose chemotherapy (with or without total body irradiation; Level of evidence I) and adult patients receiving 
radiation therapy to the head and neck (with chemotherapy, Level of evidence I, or without chemotherapy, Level of evidence II). Each guideline is outlined according to patient age group and oncologic therapy. It should be noted that not all photobiomodulation protocols are effective for the above-mentioned situations, and the clinician should refer to the full text for specific evidence-based photobiomodulation protocols [8]. Based on the current evidence, no guideline was possible for photobiomodulation therapy for: the prevention of oral mucositis in cancer patients treated with chemotherapy only (with no radiation therapy or hematopoietic stem cell transplantation); the treatment of established oral mucositis; and pediatric cancer patients.

\section{Safety}

Regarding the safety of photobiomodulation, none of the studies included in the analysis reported short- or long-term adverse events, except one cohort study, in which $15 \%$ of patients experienced an immediate transient (nonpainful) burning sensation after intra-oral $635 \mathrm{~nm}$ diode laser treatment. There is a clinical concern about the potential long-term risk of photobiomodulation therapy inducing malignant transformation, progression or recurrence, especially in head and neck cancer patients [9]. It is currently unknown whether photobiomodulation therapy directly targeted at the site of a malignant lesion has a negative impact on oncologic treatment (i.e., radiation therapy) or prognosis. Therefore, in the vast majority of studies, the photobiomodulation was not applied directly over tumor sites; this practice seems reasonable until we have a better understanding of the response of malignant or premalignant cells to photobiomodulation therapy. In two follow-up studies, an increase in the progression-free survival following photobiomodulation therapy in head and neck cancer patients treated with radiation therapy was noted $[10,11]$, likely due to the lower prevalence of oral mucositis after photobiomodulation therapy, which then allowed the completion of the planned oncologic treatment. Obviously, laser safety standards and standard precautions of infection control must be employed during photobiomodulation therapy.

\section{Adoption}

According to Rogers' Theory of Diffusion of Innovations, the adoption of a new innovation includes an interaction between the individual and the innovation itself, and comprises from five steps: acquiring information; obtaining an attitude; making a decision to adopt/reject the innovation; implementing the new innovation; and continued examination of the decision and changing the individual's behavior accordingly [12]. In regards to photobiomodulation for the prevention of oral mucositis, step 1 may be fulfilled in reading this editorial and the full paper [8].

Rogers also described five factors influencing the pace of the innovation adoption: the individual's perception of the advantage of the new innovation in comparison to the existing state; the compatibility of the innovation with existing structures; the perceived difficulty level of adopting the innovation; the testability of the new innovation; and the visibility of outcomes resulting from the innovation adoption $[13,14]$.

Practical and economic considerations may challenge and restrict the clinical adoption of the new guidelines by clinicians and institutions. These considerations include the cost of the laser device and operator time, the need for trained operators and a closed room, and any local regulation. However, the applicability of photobiomodulation therapy to cancer patients was demonstrated by Treister et al. [15], among others. Therefore, photobiomodulation therapy should be positively considered by the clinician, among the other treatments that were recommended by the MASCC/ISOO, when the clinical indication exists and the institution's facilities and health economy support the use of photobiomodulation therapy for the prevention of oral mucositis.

\section{Future directions}

The future is promising: the newly recommended protocols are based on current literature. However, future studies may show the efficacy of photobiomodulation protocols that have not yet been studied, or which may be effective in other cancer populations (i.e., pediatric patients), or in settings that were previously thought to be inefficient (i.e., extra-oral photobiomodulation and other intra-oral photobiomodulation protocols). Photobiomodulation therapy is patient-friendly and well-tolerated, especially in pediatric patients that may not be able to comply with other modalities such as mouthwashes; however, as mentioned above, there is still no recommended photobiomodulation protocol for preventing or treating oral mucositis in this population. Extra-oral photobiomodulation therapy may be more convenient for both the patient and clinician in comparison to intra-oral photobiomodulation, with the ability to treat large areas in a relatively short time. Furthermore, the intra-oral discomfort and restricted mouth opening often found in head and neck cancer patients following radiation therapy make an extra-oral approach attractive. No protocol for extra-oral photobiomodulation for preventing or treating oral mucositis has 
been formulated. Nevertheless, the new guidelines provide the prevention protocols for various indications, and more treatments may be added soon.

Being a major dose-limiting condition, as mentioned above, the prevention of oral mucositis through the new guidelines of MASCC/ISOO enables the patient to receive an optimal antiancer therapy with obvious influence on his or her prognosis, fulfilling the slogan of MASCC that declares 'Supportive care makes excellent cancer care possible'.

\section{Acknowledgments}

Y Zadik is the head of the Photobiomodulation, laser and other light section, Mucositis Study Group, of the Multinational Association of Supportive Care in Cancer and the International Society of Oral Oncology (MASCC/ISOO), and is an active member of the Cancer Supportive Care Working Group of World Association for Photobiomodulation Therapy (WALT).

\section{Financial \& competing interests disclosure}

The author has no relevant affiliations or financial involvement with any organization or entity with a financial interest in or financial conflict with the subject matter or materials discussed in the manuscript. This includes employment, consultancies, honoraria, stock ownership or options, expert testimony, grants or patents received or pending, or royalties.

No writing assistance was utilized in the production of this manuscript.

\section{References}

1. Arany PR. Craniofacial wound healing with photobiomodulation therapy: new insights and current challenges. J. Dent. Res. 95(9), 977-984 (2016).

2. Zecha JA, Raber-Durlacher JE, Nair RG et al. Low-level laser therapy/photobiomodulation in the management of side effects of chemoradiation therapy in head and neck cancer: part 2: proposed applications and treatment protocols. Support. Care Cancer 24(6), 2793-2805 (2016).

3. Sonis ST. Oral mucositis. Anticancer Drugs 22(7), 607-612 (2011).

4. Elad S, Zadik Y. Chronic oral mucositis after radiotherapy to the head and neck: a new insight. Support. Care Cancer 24(11), 4825-4830 (2016).

5. Elad S, Zadik Y, Yarom N. Oral complications of nonsurgical cancer therapies. Atlas Oral Maxillofac. Surg. Clin. North Am. 25(2), 133-147 (2017).

6. Elad S, Bowen J, Zadik Y, Lalla RV. For the mucositis study group of the multinational association of supportive care in cancer/international society of oral oncology (MASCC/ISOO). Development of the MASCC/ISOO clinical practice guidelines for mucositis: considerations underlying the process. Support. Care Cancer 21(1), 309-312 (2013).

7. Elad S. The MASCC/ISOO mucositis guidelines 2019 update: introduction to the first set of articles. Support. Care Cancer (2019) (In Press).

8. Zadik Y, Arany PR, Fregnani ER et al. For the mucositis study group of the multinational association of supportive care in cancer/international society of oral oncology (MASCC/ISOO). Systematic review and guidelines of photobiomodulation for the management of oral mucositis in cancer patients. Support. Care Cancer (2019) (In Press).

9. Sonis ST, Hashemi S, Epstein JB, Nair RG, Raber-Durlacher JE. Could the biological robustness of low level laser therapy (Photobiomodulation) impact its use in the management of mucositis in head and neck cancer patients. Oral Oncol. 54(1), 7-14 (2016).

10. Antunes HS, Herchenhorn D, Small IA et al. Long-term survival of a randomized phase III trial of head and neck cancer patients receiving concurrent chemoradiation therapy with or without low-level laser therapy (LLLT) to prevent oral mucositis. Oral Oncol. 71(1), 11-15 (2017).

11. Brandao TB, Morais-Faria K, Ribeiro ACP et al. Locally advanced oral squamous cell carcinoma patients treated with photobiomodulation for prevention of oral mucositis: retrospective outcomes and safety analyses. Support. Care Cancer 26(7), 2417-2423 (2018).

12. Rogers EM. Diffusion of innovations (4th Edition). Free Press, NY, USA (1995).

13. Rogers EM. Lessons for guidelines from the diffusion of innovations. Jt. Comm. J. Qual. Improv. 21(7), 324-328 (1995).

14. Rogers EM. Diffusion of preventive innovations. Addict. Behav. 27(6), 989-993 (2002).

15. Treister NS, London WB, Guo D et al. A feasibility study evaluating extraoral photobiomodulation therapy for prevention of mucositis in pediatric hematopoietic cell transplantation. Photomed. Laser Surg. 34(4), 178-184 (2016). 
